Infusion Therapy

Transfusion Medicine

Infusionstherapie

Transfusionsmedizin

\section{Concerning: Zeiler et al: In vitro properties of platelet concentrates. Infus Ther Transfus Med 2001;28:9-14.}

I read with interest the recent paper by Zeiler et al. entitled «In vitro properties of platelet concentrates».

The paper demonstrates adverse effects of long-term $(20 \mathrm{~h})$ platelet storage together with red blood cells and granulocytes in buffy coats. Consequently, the study confirms earlier reports [1].

The authors conclude that the clinical relevance of their findings has to be proven. The conclusion seems to be open for criticism from an ethical point of view. It is possible that the disadvantage with $20 \mathrm{~h}$ buffy coat storage actually has clinical relevance. Consequently, the ethical approach must be to avoid long-term buffy coat storage until proven that it does not cause clinical damage.

Järemo P., Linköping

\section{References}

1 Järemo R, Shaba A, Kutti J: Some storage characteristics of buffy coats used for the preparation of platelet concentrates. Ann Hematol 1992;65:269-273.

\section{Zu: Kretschmer V, Karger R: Neue Richtlinien zur Gewinnung von Blut und Blutbestandteilen und zur Anwendung von Blutprodukten (Hämotherapie) - Änderun- gen, Interpretation und Kommentar. Infus Ther Transfus Med 2001;28:24-43, 82-94.}

Für die ausführliche Kommentierung und Interpretation der neuen Richtlinien den Autoren einen ganz herzlichen Dank. Trotz aller Beschäftigung mit den Richtlinien bleiben Möglichkeiten verschiedener Interpretationen und auch offene Fragen. Gerne möchte ich die Gelegenheit wahrnehmen, meine Fragen und anderslautenden Interpretationen zur Diskussion zu stellen.

Zum Thema «Eigenblut mit positiven Infektionsmarkern» finde ich erwähnenswert, dass eine Weitergabe des Befundes über den Infektionsstatus des Patienten schriftlich an den transfundierenden Arzt erfolgen soll (4.6.3: «Bei der Ausgabe der betreffenden Produkte ist der transfundierende Arzt über den infektiösen Status schriftlich zu informieren.») allerdings nur nach Einwilligung des Patienten (2.7.1.3: «... Die verantwortliche ärztliche Person der Einrichtung hat den Patienten und nach Einwilligung dessen transfundierenden Arzt unverzüglich über den anlässlich der (Eigenblut-)Entnahme gesichert festgestellten Infektionsstatus zu unterrichten ...»). Es ist sicher sinnvoll, auch diese Einwilligung schriftlich zu dokumentieren. Eine Transfusion von Eigenblut mit positiven Infektionsparametern ist demnach nur möglich, wenn der Patient auch gleichzeitig mit der Weitergabe seiner Befunde an den transfundierenden Arzt einverstanden ist.

Ebenfalls bemerkenswert finde ich, dass in den neuen Richtlinien eine Eigenblut-Methode beschrieben wird, die dem Patienten in höchstem Maße Schaden zufügen kann (2.7.3: «Autologe Direkt-Retransfusion: ... Bei dieser Form der Retransfusion von postoperativ gesammeltem Drainageblut besteht abhängig vom transfundierten Volumen die Gefahr einer massiven Gerinnungsaktivierung und Bakteriämie.»). Da die Richtlinien der Patientensicherheit dienen sollen, ist meiner Meinung nach die Beschreibung einer solchen Methode obsolet.

Leider unklar bleibt, in welcher Form die Dokumentation von Notfällen zu erfolgen hat (4.2.5.10: «... Notfälle und die Abweichung von den Richtlinien sind schriftlich zu dokumentieren.»). Genügt z.B. das Narkoseprotokoll, in dem Aufzeichnungen über Kreislaufverhältnisse des Patienten, Laborergebnisse wie Blutbild, Blutgase, Gerinnung usw. gemacht werden?

$\mathrm{Zu}$ dem Thema Anwärmen (4.3.3: «... Das Anwärmen von Blutkomponenten (max. $37^{\circ} \mathrm{C}$ ) beschränkt sich ...») kommentieren die Autoren, dass die Anwärmgeräte höhere Temperaturen als $37^{\circ} \mathrm{C}$ aufweisen dürfen, wenn sichergestellt ist, dass die Höchsttemperatur von $37^{\circ} \mathrm{C}$ im Präparat nicht überschritten wird. Da dieser Unterschied ja erhebliche Bedeutung haben kann, interessiert es mich, ob es dazu verbindliche Vereinbarungen gibt.

Anlass zu Missverständnissen gibt unter Umständen der Punkt 2.2.2: «Von der Blutspende zeitlich begrenzt zurückzustellen sind Personen ... nach Empfangen eines Transplantates humanen Ursprungs (außer Kornea und Dura mater) für 6 Monate, ...». Dies könnte so interpretiert werden, als ob Empfänger von Kornea- und Dura-mater-Transplantaten nicht von der Blutspende zurückzustellen sind und spenden dürfen. Angebracht wäre hier ein deutlicher Hinweis auf Punkt 2.2.1: «Als Blutspender auf Dauer auszuschließen sind Personen ..., die Dura-mater- bzw. Kornea-Transplantate erhalten haben, ...».

\begin{tabular}{ll}
\hline KARGER & @ 2001 S. Karger GmbH, Freiburg \\
$\begin{array}{l}\text { Fax }+497614520714 \\
\begin{array}{l}\text { E-mail Information@Karger.de } \\
\text { www.karger.com }\end{array}\end{array}$ & Accessible online at: \\
www.karger.com/journals/iut
\end{tabular}

\section{KARGER}

www.karger.com 
Einer Interpretation durch die Autoren möchte ich widersprechen. In dem im Kommentar ganz wiedergegebenen Abschnitt über «Transport und Lagerung in der Einrichtung der Krankenversorgung» (4.1) wird von den Autoren der Begriff «Anwender» mal als periphere Station, OP, Ambulanz usw. interpretiert, bei der «Lagerung von Blutpräparaten beim Anwender» dann aber als Blutdepot gedeutet. Ich bin der Meinung, dass auch hier tatsächlich der Anwender auf der Station, im OP, in der Ambulanz usw. gemeint ist. Werden dort, wie z.B. bei uns auf einigen Intensivstationen, durchaus für mehrere Tage Blutpräparate gelagert (weil z.B. der Weg vom Blutdepot sehr weit ist), so sollten die dafür vorgesehenen Kühleinrichtungen durchaus den unter Abschnitt 3.3 geforderten Kriterien entsprechen.

Dies als ergänzende Anmerkungen und Diskussionsbeitrag zu den neuen Richtlinien.

S. Quentin, Göttingen

\section{Antwort der Autoren}

Wir möchten uns ausdrücklich für den Diskussionsbeitrag zu unserem Richtlinienkommentar bedanken, da das Angebot dieser Zeitschrift, kontroverse Standpunkte öffentlich zu diskutieren, leider so wenig angenommen wird.

Wir stimmen der Autorin des Leserbriefes in fast allen angesprochenen Punkten zu. Notfälle sollten einerseits, wie angesprochen, aus der Patientenakte, z.B. dem Narkoseprotokoll, abgeleitet werden können. Darüber hinaus muss aber auch die Blutanforderung den medizinisch begründeten Notfall ausweisen, damit sich daraus das weitere Vorgehen im Labor begründen lässt. Dazu bedarf es lediglich der vom zuständigen Arzt unterschriebenen Notfallanforderung. Im Übrigen sollte man gerade in dieser kritischen Situation keine unnötige Bürokratie walten lassen.

Die Richtlinien wurden bezüglich ihrer Aussage zum Bluterwärmen immer wieder dahingehend missverstanden, dass die Anwärmtemperatur des Gerätes nicht über $37^{\circ} \mathrm{C}$ liegen darf. Entscheidend ist, dass die Erythrozyten nicht so stark erwärmt werden, dass sie hämolysiert werden. Bei Erwärmen von Blut auf maximal $38^{\circ} \mathrm{C}$ ist das sicher als gegeben anzunehmen. Die AABB-Standards von 1996 haben sogar eine Temperatur von $42{ }^{\circ} \mathrm{C}$ als Höchstgrenze festgelegt [1]. Im Hinblick auf den Wärmeverlust durch Beutelfolie bzw. Plas- tikschläuche sowie abhängig von Kontaktfläche und Anwärmzeit muss die vom Blutwärmer entwickelte Temperatur entsprechend höher sein, als die in der Blutkonserve angestrebte Temperatur. Die American Society for Testing and Materials (ASTM) bereitet derzeit in einem Subkomitee eine Standardspezifikation für Blutwärmer vor, bei der die erlaubte Höchsttemperatur abhängig von der Einwirkdauer ist: $46^{\circ} \mathrm{C} 1 \mathrm{~h}$, $47^{\circ} \mathrm{C} 30 \mathrm{~min}, 48{ }^{\circ} \mathrm{C} 10 \mathrm{~min}, 48,6{ }^{\circ} \mathrm{C} 2 \mathrm{~min}$ [2]. Auch bei uns sollten Anwärmgeräte erst wissenschaftlich geprüft und die daraus abzuleitende Anwendung definiert werden. «Verbindliche Vereinbarungen» im Sinne von Frau Quentin stellen die Richtlinien dar und werden darüber hinaus durch die TÜVZulassung der Geräte geschaffen.

Auch bezüglich der Zwischenlagerung von Blutprodukten in der Klinik widersprechen wir der Autorin des Leserbriefes nicht. Für Blutkonserven, die peripher wie in einem Depot über längere Zeit gelagert werden, und gegebenenfalls auch wieder in das zentrale Depot zurück genommen werden sollen, müssen selbstverständlich die Lagerungsbedingungen wie beim Hersteller erfüllt sein. Dies wurde in unserem Kommentar für OP-Kühlschränke dargestellt [3]. Davon ist nur abzuweichen, wenn die Blutkonserven innerhalb von wenigen Stunden sicher transfundiert werden. Die Richtlinien führen ausdrücklich aus, dass in der Regel Blutkonserven bei Abgabe an den Anwender zur unmittelbaren Anwendung bestimmt sind, und für diesen Fall halten wir keine besonderen Kühlaggregate für die Zwischenlagerung und den Transport für erforderlich. Die Abgabe von Blutkonserven an periphere Depots, z.B. auf Intensivstationen, beinhaltet, vor allem bei mehrtägiger Lagerung, noch andere Risiken (vor allem verzögerte hämolytische Transfusionsreaktionen, unnötig große Blutdepots mit schlechterer Qualität der Blutkonserven und vermehrtem Verfall), weshalb vor der Einrichtung solcher peripheren Depots ausdrücklich zu warnen ist.

\section{Kretschmer, R. Karger, Marburg}

\section{Literatur}

1 American Association of Blood Banks (AABB): Standards for blood banks and transfusion services. 17th ed. Bethesda, MD, AABB, 1996.

2 American Society for Testing and Materials, Subcommittee ASTM F29-19-02; Blood and Infusion Warmer. Draft 10, 2001.

3 Kretschmer V, Karger R: Neue Richtlinien zur Gewinnung von Blut und Blutbestandteilen und zur Anwendung von Blutprodukten (Hämotherapie) - Änderungen, Interpretationen und Kommentare (Teil 2). Infus Ther Transfus Med 2001;28:82-94. 